\title{
ENERGY, MACRONUTRIENTS AND DIETARY FIBRE INTAKE AMONG ADULTS IN NORTH MACEDONIA
}

\author{
Igor Spiroski ${ }^{1,2}$, Marina Nikolić ${ }^{3}$, Mihail Kochubovski ${ }^{1,2}$, Mirjana Gurinović ${ }^{4}$, Gordana Ristovska ${ }^{1,2}$, Agnes Kadvan $^{5}$ \\ ${ }^{1}$ Institute of Public Health, Skopje, North Macedonia \\ ${ }^{2}$ Faculty of Medicine, Ss. Cyril and Methodius University, Skopje, North Macedonia \\ ${ }^{3}$ European Food Safety Authority, Parma, Italy \\ ${ }^{4}$ Centre of Research Excellence in Nutrition and Metabolism, Institute for Medical Research, University of Belgrade, Belgrade, Serbia \\ ${ }^{5}$ Capacity Development Network in Nutrition in Central and Eastern Europe, CAPNUTRA, Belgrade, Serbia
}

\section{SUMMARY}

Objective: The present research aimed to estimate macronutrients intake among adults in North Macedonia and to identify their predominant food sources.

Methods: Within this cross-sectional study dietary data were collected using repeated $24 \mathrm{~h}$ recall interviews. Nationwide, adults aged $18+$ were recruited. Dietary data, anthropometric measures and socio-demographic characteristics were available for 496 participants. Nutrient intake was analyzed using the Balkan Food Composition Database and Diet Assess and Plan platform. Macedonian dietary guidelines were used for estimation of inadequacy.

Results: Significant differences in macronutrients intake are noticed between age groups within certain socio-demographic factors. Total sugar intake was significantly higher among young adult females comparing with those older than 25 years $(p=0.049)$. Young urban females have significantly higher fats intake than older participants $(p=0.038)$. Higher total daily energy, proteins, fats and carbohydrates intake $(p=0.033$, $p=0.043, p=0.032, p=0.042$, respectively) was noticed among young urban males when compared to older ones. Only dietary fibre intake was higher among older urban males $(p=0.030)$. Univariate linear regression models showed that obese participants had significantly higher relative proteins intake comparing with those having BMI within recommended range $(p=0.024, \beta=1.21)$. Relative carbohydrates intake was significantly lower among males $(p=0.018, \beta=-2.077)$ and among highly educated participants $(p=0.018, \beta=-4.304)$. Participants with tertiary education had higher relative fats intake $(p=0.012, \beta=4.213)$.

Conclusion: Macronutrients intake of adults should be improved. There is higher intake of dietary fats and need for an increase of complex carbohydrates intake, particularly dietary fibre. Findings of this survey should be used in shaping, fine-tuning and implementing food and nutrition policies that will stimulate healthier diets for prevention of diet related non-communicable diseases.

Key words: macronutrients, dietary habits, nutritional assessment, food consumption survey

Address for correspondence: I. Spiroski, Institute of Public Health, 50. Divizija 6, 1000 Skopje, North Macedonia. E-mail: i.spiroski@iph.mk

https://doi.org/10.21101/cejph.a5345

\section{INTRODUCTION}

Dietary risk factors are proven to be the leading global contributors both in deaths and in Disability Adjusted Life Years (DALYs). High body mass index (BMI) is the leading risk factor in many countries along with the high blood pressure, another health risk closely related with nutrition and dietary habits of the population (1). Robust research data show correlation between diet and most of the leading non-communicable diseases (NCDs) and also beneficial health effects when dietary patterns are improved (2). The Republic of North Macedonia (MKD) is no exception when both leading risk factors and NCDs are compared to other developed or developing countries. NCDs are leading cause for premature dying in Macedonian population, attributing for most of the Years of Life Lost (YLL). In terms of DALYs in MKD, dietary risks and high systolic blood pressure are the leading risk factors (3). Cardiovascular diseases (CVD) and cancers account for around $80 \%$ of all deaths. Additionally, there is high rate of fatal outcomes related to type 2 diabetes mellitus in MKD, accounting for $4 \%$ of all deaths. Dietary guidelines for the population of MKD were developed by the Institute of Public Health (IPH) and were formally endorsed by the Government, in 2014 (4). The Guidelines include the Dietary Intakes (DRIs) for nutrients and qualitative guidelines for healthy eating patterns.

Nutritional epidemiology, as relatively new area of research, is growing and expanding, and the need for data to implement science into policy is inevitable (5). Comprehensive population data about the food consumption of adults in MKD has not been collected and analyzed until our survey was initiated. Availability of comprehensive food consumption data in Southern Europe is also very limited. Available data suggest that the countries in the region share similar dietary patterns (6). Consequently, there was a need for population based national food consumption survey, both for research and policy purposes. The data collection for the first Food Consumption Survey (FCS) in MKD took place during the period April-June 2015 with the objective to assess the food 
and drink consumption among the Macedonian adults. The study was initiated to assess the baseline situation having in mind that no nationally representative data on food consumption of adults existed in MKD. The aim of this paper is to analyze the energy, macronutrients and fibre intake, and to identify the predominant food sources for it in the diet of the adult population in MKD. Additionally, the study investigates the adherence of the population to the existing national dietary recommendations.

\section{MATERIALS AND METHODS}

\section{Sampling}

Sampling frame was based on the data of telephone (book) database, census of population from 2002, vital statistics and the migrations data, as well as estimates of population and households, annually provided by the State Statistical Office (7). It was a 2-staged stratified combined probability sample through sampling households by simple random sampling without replacement (SRSWOR) in the first stage and household's member according to quota criteria in the second one. Stratification was done according to type of settlement (urban or rural), four geoeconomical regions, age groups and gender. Allocation of the sample by stratum was proportional to the size of the stratum. $95 \%$ confidence interval for incidence of $50 \%$ on sample size of 1,000 was $\pm 3.2 \%$. Automatic control of procedure, interactive control of consistency and logical control of collected data was performed in $100 \%$ of cases.

Only one member of the household aged $18+$ was interviewed. The interviews with the selected members of the households lasted for approximately 30 minutes on average. Anthropometric data were also collected based on self-reported information on height and weight. Socio-demographic characteristics of the study sample are presented in Table 1 . Total of 219 men and 277 women were involved in the survey. Average age of respondents was 46.0 (47.8 for men and 45.2 for women). Age groups breakdown is as follows: 80 respondents were $18-24$ years old; 319 were at the age group of 25-64 years; and 87 were older than 65. Regarding their place of living, 322 are living in urban and 174 in rural areas.

\section{Dietary Assessment}

Dietary data were collected using repeated $24 \mathrm{~h}$ recall phone interviews conducted by trained interviewers. Data was collected for a representative sample of 496 respondents older than 18 years for two non-consecutive days. Time difference between the two interviews was approximately four weeks. The respondents were asked to report the complete consumption of food and beverages from the previous day (a day starting at midnight before the day of the interview). According to the methodology, interviewers were trained and instructed to obtain the quantification of food and drinks consumed in grams, using household measures. Interviewers also collected the data about recipes and their preparation and cooking methods. Using multiple pass technique, interviewers were able to capture food items that participants usually forget to report such as snacks, sweets, spices and liquids.

\section{Nutrient Analysis}

Macronutrients intake was estimated using the Balkan Food Composition Database (8). Total daily intake of macronutrients and contribution of particular food groups to total energy from carbohydrates, proteins and fats was calculated using the Diet Assess and Plan, the dietary assessment software tool validated in national and regional consumption studies and evaluated in a European Food Safety Authority (EFSA) project (9). The average daily macronutrients intake was calculated on individual level. Contribution of 13 food groups to average relative macronutrients intake was calculated.

\section{Comparison According to Population Goals}

Inadequacy of macronutrients intake was analyzed using existing population goals for MKD (4). For residents of MKD total carbohydrates intake should account for $45-65 \%$ of total energy, total protein intake for $10-35 \%$, and the adequate intake of fats for $20-35 \%$ of total energy intake. We have also analyzed inadequacy for total daily fats intake for upper limit of $30 \%$ of total energy intake according to the WHO/FAO population goals in order to see the difference among participants who had inadequate intake according to these two versions of recommendations. Inadequacy for fatty acids was calculated according to the recommendations of the European Heart Network (10). Percentage of individuals in the population who met the dietary recommended target for macronutrients was calculated for four socio-demographic categories that have been analyzed: age, gender, education, and type of settlement. Nutritional adequacy was also analyzed according to the BMI status.

\section{Statistical Analysis}

Mean and standard deviation were calculated for BMI, macronutrients and their components by age, gender and type of urbanization. Chi-square test was performed to check for the association between socio-demographic factors and BMI status as well as macronutrients inadequacy. T-test was used to assess the gender differences and type of urbanization while one-way analysis of variance (ANOVA) was used to assess difference within factors that have more than two categories. When statistically significant effects were identified, comparisons of means were further examined using Bonferroni correction to ascertain which specific means differed. For variables that were not normally distributed the Mann-Whitney and Kruskal-Wallis non-parametric test were used for comparison between two or more groups. Univariate linear regression models were used to explore the association between relative macronutrients contribution to energy intake and prediction factors. Value of $\mathrm{p}<0.05$ was considered statistically significant. All statistical analyses were performed using SPSS software package for Windows (version 20). Tables and figures were created using Microsoft Excel spreadsheets (version 1997 SR-2, Microsoft Corporation, Redmond, WA).

\section{RESULTS}

Table 2 presents results on anthropometric data. BMI was calculated based on self-reported height and weight and analyzed 
by socio-demographic factors. We have found that females had significantly lower BMI than males and adults younger than 25 significantly lower than older ones. Participants were classified as underweight $\left(\mathrm{BMI}<18.5 \mathrm{~kg} / \mathrm{m}^{2}\right)$, weight within recommended range (BMI 18.5-24.9 kg/m²), overweight (BMI 25.0-29.9 kg/

Table 1. Demographic characteristics of the sample $(N=496)$

\begin{tabular}{|l|c|c|c|}
\hline \multicolumn{5}{|c|}{$\begin{array}{c}\text { Males (n=219) } \\
\mathbf{n}(\%)\end{array}$} & $\begin{array}{c}\text { Females (n=277) } \\
\mathbf{n}(\%)\end{array}$ & $\begin{array}{c}\text { Total } \\
\mathbf{n}(\%)\end{array}$ \\
\hline Age & $32(14.6)$ & $48(17.3)$ & $80(16.1)$ \\
\hline $18-24$ & $141(64.4)$ & $178(64.3)$ & $319(64.3)$ \\
\hline $25-64$ & $46(21.0)$ & $51(18.4)$ & $97(19.6)$ \\
\hline$\geq 65$ & $138(63.0)$ & $184(66.4)$ & $322(64.9)$ \\
\hline Type of settlement & $81(37.0)$ & $93(33.6)$ & $174(35.1)$ \\
\hline Urban & $12(5.5)$ & $23(8.3)$ & $35(7.1)$ \\
\hline Rural & $134(61.2)$ & $171(61.7)$ & $305(61.5)$ \\
\hline Education & $73(33.3)$ & $83(30.0)$ & $156(31.5)$ \\
\hline Primary & $1(0.5)$ & $17(6.1)$ & $18(3.6)$ \\
\hline Secondary & $80(36.5)$ & $127(45.8)$ & $207(41.7)$ \\
\hline Tertiary & & & $184(37.1)$ \\
\hline BMl & $96(43.8)$ & $88(31.8)$ & $97(19.6)$ \\
\hline Underweight & $42(19.2)$ & $45(16.2)$ & \\
\hline $\begin{array}{l}\text { Weight within } \\
\text { recommended } \\
\text { range }\end{array}$ & & & \\
\hline Overweight & \multicolumn{3}{|l}{} \\
\hline Obese & \multicolumn{5}{|l}{} \\
\hline
\end{tabular}

$\left.\mathrm{m}^{2}\right)$, and obese $\left(>30 \mathrm{~kg} / \mathrm{m}^{2}\right)$. Significantly more females were underweight and had weight within recommended range, comparing to males who were more overweight and obese. Distribution of participants in BMI categories is also affected by age groups. There were significantly more underweight participants among young adults comparing to those older than 25 while more than $40 \%$ of adults older than 25 were overweight. The highest prevalence of overweight and obese participants was observed in the elderly group. Also, $40 \%$ of primarily educated participants were obese. Table 3 presents average daily intake of energy, macronutrients and fibre, as well as contribution of macronutrients to the total daily energy intake by age, gender and urbanization groups. We have examined effect of age factor within the gender and urbanization type and found that among rural females there was significant difference in relative sugar intake between age groups $(p=0.049)$. On the other side, among urban females, age factor affected total fats intake $(p=0.038)$. Age factor has shown a huge impact on urban males. Significant difference was found between age groups for total daily energy, proteins, fats and carbohydrates intake ( $p=0.033, p=0.043, p=0.032, p=0.042$, respectively). Relative intake of fats was also affected by age factor within this group $(\mathrm{p}=0.010)$. It is noteworthy to mention that adults younger than 25 years have higher intake of macronutrients than older participants except for the relative fats intake where the highest average relative intake was noticed among adults aged 25 to 65 . It is interesting that age factor affects fibre intake too, but the trend was opposite $(p=0.030)$. Analyzing differences in macronutrients intake with respect to urbanization (within gender, excluding age factor) we have found that rural females had lower total sugar intake comparing with those living in urban areas. There were no such differences among men living in different urbanization settings. When analyzing macronutrients intake among rural

Table 2. Distribution of participants according to their BMI status and SES factors $(N=496)$

\begin{tabular}{|c|c|c|c|c|c|c|c|}
\hline & $\begin{array}{c}\text { BMI } \\
(\text { mean } \pm S D)\end{array}$ & \multirow[t]{2}{*}{ p-value* } & $\begin{array}{c}\text { Underweight } \\
\mathrm{n}(\%)\end{array}$ & $\begin{array}{c}\text { Weight within } \\
\text { recommended } \\
\text { range } \\
\mathrm{n}(\%)\end{array}$ & $\begin{array}{l}\text { Overweight } \\
\text { n (\%) }\end{array}$ & $\begin{array}{l}\text { Obese } \\
\mathrm{n}(\%)\end{array}$ & \multirow[t]{2}{*}{$p$-value* } \\
\hline & $25.8 \pm 4.8$ & & $18(3.6)$ & $207(41.7)$ & $184(37.1)$ & $87(17.5)$ & \\
\hline \multicolumn{8}{|l|}{ Gender } \\
\hline Female & $25.4 \pm 5.3$ & \multirow{2}{*}{$<0.001$} & $17(6.1)$ & $127(45.8)$ & $88(31.8)$ & $45(16.2)$ & \multirow{2}{*}{$<0.001$} \\
\hline Male & $26.3 \pm 3.9$ & & $1(0.5)$ & $80(36.5)$ & $96(43.8)$ & $42(19.2)$ & \\
\hline \multicolumn{8}{|l|}{ Age } \\
\hline $18-24$ & $21.8 \pm 3.6$ & \multirow{3}{*}{$<0.001$} & $11(13.8)$ & $53(66.3)$ & $13(16.3)$ & $3(3.8)$ & \multirow{3}{*}{$<0.001$} \\
\hline $25-64$ & $26.4 \pm 4.6$ & & $7(2.2)$ & $123(38.6)$ & $130(40.8)$ & $59(18.5)$ & \\
\hline$\geq 65$ & $27.2 \pm 4.4$ & & $0(0.0)$ & $31(32.0)$ & 41 (42.3) & $25(25.8)$ & \\
\hline \multicolumn{8}{|c|}{ Type of settlement } \\
\hline Rural & $26.2 \pm 5.6$ & \multirow{2}{*}{ ns } & $7(4)$ & $70(40.2)$ & $59(33.9)$ & $38(21.8)$ & \multirow{2}{*}{ ns } \\
\hline Urban & $25.6 \pm 4.3$ & & $11(3.4)$ & $137(42.5)$ & $125(38.8)$ & $49(15.2)$ & \\
\hline \multicolumn{8}{|l|}{ Education } \\
\hline Primary & $27.5 \pm 5.9$ & \multirow{3}{*}{ ns } & $1(2.9)$ & $13(37.1)$ & $7(20.0)$ & $14(40.0)$ & \multirow{3}{*}{0.024} \\
\hline Secondary & $25.6 \pm 4.6$ & & $12(3.9)$ & $130(42.6)$ & $117(38.4)$ & $46(15.1)$ & \\
\hline Tertiary & $25.7 \pm 4.8$ & & $5(3.2)$ & $64(41.0)$ & $60(38.5)$ & $27(17.3)$ & \\
\hline
\end{tabular}

ns - not significant; *Mann Whitney test was used for comparison between rural and urban as well as between females and males while Kruskal-Wallis test was used for comparison between different age groups; ${ }^{* *}$ Chi-square test was used for comparison between BMI status categories and SES factors. 


\begin{tabular}{|c|c|c|c|c|c|c|c|c|c|c|c|c|c|c|c|c|c|c|c|c|c|c|c|c|c|c|}
\hline & & 6 & $\begin{array}{l}\bar{\delta} \\
\dot{0} \\
\dot{v}\end{array}$ & $\begin{array}{l}\bar{\delta} \\
\dot{D} \\
\dot{v}\end{array}$ & & $\begin{array}{l}\bar{\delta} \\
\dot{0} \\
\mathrm{v}\end{array}$ & & $\begin{array}{l}\bar{\delta} \\
\dot{0} \\
\bar{v}\end{array}$ & & & $\begin{array}{l}\text { ₹ै } \\
\circ\end{array}$ & $\begin{array}{l}\bar{\delta} \\
\dot{0} \\
\end{array}$ & & "a & & $\begin{array}{l}\bar{\delta} \\
\dot{0} \\
\mathrm{v}\end{array}$ & $\begin{array}{l}\bar{\delta} \\
\dot{0} \\
\bar{v}\end{array}$ & & $\begin{array}{l}\bar{\delta} \\
\dot{0} \\
v\end{array}$ & & $\begin{array}{l}\bar{\delta} \\
\dot{0} \\
\bar{v}\end{array}$ & & $\begin{array}{l}\bar{\delta} \\
\dot{0} \\
\dot{v}\end{array}$ & & $\begin{array}{l}\bar{\delta} \\
\dot{Q} \\
\mathrm{v}\end{array}$ & \\
\hline & $\begin{array}{l}\widehat{\widehat{\infty}} \\
\stackrel{11}{\leq}\end{array}$ & ஜ & $\underset{\mp}{\sim}$ & 孞 & $\stackrel{\mid}{+}$ & $\underset{\dot{s}}{\dot{S}}$ & $\stackrel{m}{\circ}$ & 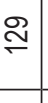 & $\stackrel{\digamma}{\risingdotseq}$ & 怘 & $\begin{array}{l}0 \\
0 \\
0\end{array}$ & $\stackrel{\vec{F}}{\rightleftarrows}$ & & 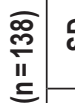 & के & $\widehat{\vec{c}}$ & $\mathscr{?}$ & $\stackrel{m}{\sim}$ & $\widehat{\vec{y}}$ & 0 & $\cong$ & $\hat{\sigma}$ & 每 & นี้ & $\stackrel{\sim}{\sim}$ & \\
\hline & 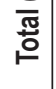 & 胥 & $\stackrel{\mid}{\stackrel{0}{F}}$ & ָ̊. & $\stackrel{\curvearrowright}{\digamma}$ & $\tilde{8}$ & $\begin{array}{l}\infty \\
\stackrel{\rho}{.}\end{array}$ & $\stackrel{0}{m}$ & 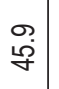 & 总 & \begin{tabular}{c|}
$\infty$ \\
$\infty$
\end{tabular} & $\stackrel{\infty}{\underset{N}{\mathbf{N}}}$ & & 焉 & $\begin{array}{l}\underset{\varpi}{\mathbb{d}} \\
\sum\end{array}$ & $\stackrel{\circ}{\circ}$ & 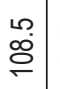 & 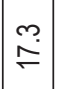 & $\tilde{\check{~}}$ & مْ & $\stackrel{̊}{\sim}$ & 每 & | & $\stackrel{\nabla}{\infty}$ & $\underset{\varpi ָ}{~}$ & \\
\hline & & 2 & $\mathscr{c}$ & $\stackrel{\mathscr{c}}{\mathbf{2}}$ & $\stackrel{\mathscr{c}}{\simeq}$ & $\stackrel{\mathscr{c}}{\mathbf{c}}$ & 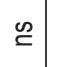 & $\stackrel{g}{c}$ & 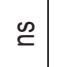 & $\mathscr{I}$ & 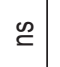 & $\mathscr{C}$ & & & & $\begin{array}{l}\widetilde{O} \\
0 \\
0\end{array}$ & $\stackrel{\mathscr{c}}{\check{2}}$ & $\mathscr{c}$ & $\begin{array}{l}\widetilde{o} \\
\tilde{O} \\
\text {. }\end{array}$ & $\bar{\delta}$ & $\begin{array}{l}\text { ₹̛ } \\
\stackrel{0}{0}\end{array}$ & 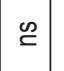 & $\stackrel{\infty}{c}$ & 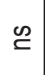 & ס̊: & \\
\hline & $\underset{\nabla}{\sigma}$ & के & $\stackrel{m}{m}$ & 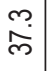 & $\stackrel{\infty}{\stackrel{m}{~}}$ & $\begin{array}{l}0 \\
\dot{q} \\
\dot{q}\end{array}$ & $\stackrel{\stackrel{\rightleftarrows}{F}}{F}$ & $\cong$ & $\stackrel{\rho}{F}$ & 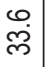 & $\hat{i}$ & $\simeq$ & & న్ల & क & $\begin{array}{l}\infty \\
\dot{m}\end{array}$ & ஓ् & $\stackrel{\infty}{\infty}$ & 守 & $\bar{\infty}^{-}$ & ิ & فم & $\begin{array}{l}\mathscr{Q} \\
\stackrel{\sim}{\sim}\end{array}$ & ָึ & $\stackrel{\infty}{\simeq}$ & \\
\hline & 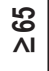 & $\mid$\begin{tabular}{l|}
$\mathbf{c}$ \\
$\stackrel{\mathbf{w}}{\Sigma}$ \\
$\Sigma$
\end{tabular} & $\stackrel{?}{\circ}$ & 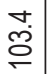 & $\neq$ & $\infty$ & 守 & శ్లి & $\overline{\widetilde{F}}$ & 管 & o) & 灾 & & $\begin{array}{l}\text { 怘 } \\
\text { 人1 }\end{array}$ & 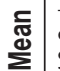 & 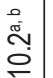 & $\begin{array}{l}\infty \\
\\
\\
\end{array}$ & $\approx$ & 它 & $\begin{array}{l}\stackrel{0}{0} \\
\stackrel{0}{\sim} \\
\sim\end{array}$ & 疍 & $\mid \begin{array}{c}m \\
\substack{q \\
o}\end{array}$ & $\begin{array}{l}1 \\
i \infty \\
i \\
i\end{array}$ & $\stackrel{\infty}{\infty}$ & i্d: & \\
\hline & $\begin{array}{l}\widehat{\tilde{n}} \\
\text { II }\end{array}$ & क् & $\stackrel{m}{\sim}$ & $\begin{array}{l}8 \\
\infty \\
\infty \\
\infty \\
\infty\end{array}$ & $\stackrel{m}{\rightarrow}$ & is & $\check{\circ}$ & $\stackrel{\mathbb{N}}{\sim}$ & $=$ & 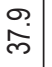 & $\stackrel{\sim}{\sim}$ & $\stackrel{\nabla}{\rightleftarrows}$ & & $\begin{array}{c}\widehat{0} \\
\text { o } \\
\text { II }\end{array}$ & क & 吕 & 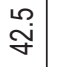 & $\stackrel{\sim}{f}$ & 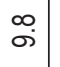 & ळे & 훙 & @. & $\begin{array}{l}0 \\
\stackrel{0}{\sim} \\
\sim\end{array}$ & نे & $\hat{\sigma}$ & \\
\hline & 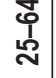 & 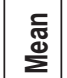 & $\stackrel{\infty}{\risingdotseq}$ & 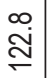 & 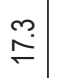 & $\begin{array}{l}\infty \\
\infty \\
\infty\end{array}$ & | & స్లి & $\begin{array}{l}\tilde{y} \\
\tilde{q}\end{array}$ & 点 & $\stackrel{\nabla}{\infty}$ & $\stackrel{\mathscr{\sim}}{\tilde{N}}$ & & $\begin{array}{l}\mathbf{0} \\
\text { 1 }\end{array}$ & 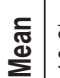 & $\begin{array}{l}\text { लि: } \\
\stackrel{0}{0}\end{array}$ & 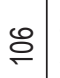 & $\stackrel{\overbrace{}}{\stackrel{2}{=}}$ & 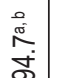 & $\frac{⿱ 乛}{\dot{f}}$ & 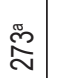 & $\ddot{\forall}$ & $\begin{array}{l}\sigma \\
\dot{q} \\
\dot{q}\end{array}$ & $\infty$ & $\begin{array}{l}\text {. } \\
\stackrel{\sim}{\circ}\end{array}$ & \\
\hline & $\underset{\nabla}{\vec{F}}$ & क & $\stackrel{+}{\forall}$ & 字 & (5) & 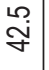 & 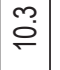 & En & $\stackrel{\text { po }}{=}$ & స్. & ما & 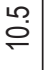 & & $\stackrel{\bar{\sigma}}{=}$ & क & $\hat{m}$ & 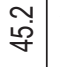 & $\overline{\text { is }}$ & $\hat{\dot{q}}$ & రె & 흐 & से & $\stackrel{\infty}{\stackrel{\sim}{j}}$ & $\dot{+}$ & $F$ & \\
\hline & $\begin{array}{l}\text { స్ } \\
\stackrel{0}{\sim}\end{array}$ & 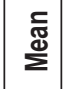 & $\stackrel{\leftrightarrow}{F}$ & $\stackrel{\stackrel{\sim}{\circ}}{\stackrel{\circ}{\sim}}$ & $\stackrel{0}{\circ}$ & $\stackrel{m}{g}$ & 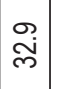 & $\stackrel{\leftrightarrow}{\sim}$ & 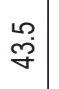 & 管 & $\widehat{\infty}$ & $\stackrel{\Sigma}{\stackrel{2}{F}}$ & & \begin{tabular}{l|}
\multirow{2}{*}{} \\
$\stackrel{0}{=}$
\end{tabular} & 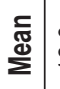 & $\begin{array}{l}\stackrel{⿰}{\infty} \\
\stackrel{\sim}{\simeq}\end{array}$ & స్. & $\stackrel{\stackrel{\circ}{r}}{=}$ & $\stackrel{\frac{\sigma}{\circ}}{\circ}$ & 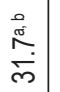 & 惫 & $\stackrel{\leftrightarrow}{\dot{J}}$ & $\widehat{\tilde{i}}$ & $\infty$ & 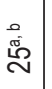 & \\
\hline & & 芝 & $\cong$ & $\stackrel{\mathscr{c}}{\simeq}$ & $\stackrel{\mathscr{c}}{\simeq}$ & $\stackrel{\infty}{c}$ & $\mathscr{c}$ & 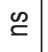 & $\stackrel{\text { உ }}{\subseteq}$ & סु & $\stackrel{2}{\simeq}$ & $\stackrel{\infty}{c}$ & & 范 & & $\stackrel{D}{c}$ & $\stackrel{\text { D }}{\simeq}$ & $\mathscr{L}$ & Ф & $\stackrel{\infty}{\simeq}$ & $\stackrel{\infty}{\simeq}$ & $\mathscr{L}$ & $\mathscr{2}$ & $\stackrel{\infty}{c}$ & $\stackrel{2}{\simeq}$ & \\
\hline & ભু & ㅇ & $\widetilde{\infty}$ & 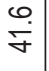 & $\stackrel{m}{\sim}$ & 年 & $\begin{array}{l}\infty \\
\infty\end{array}$ & के & ه̊ & $\begin{array}{l}\stackrel{\sim}{s} \\
\stackrel{m}{*}\end{array}$ & $\stackrel{m}{\sim}$ & ゙ָ & & $\underset{\mathbf{F}}{\stackrel{0}{=}}$ & क & $\stackrel{m}{m}$ & $\begin{array}{c}0 \\
\stackrel{9}{m} \\
\dot{m}\end{array}$ & $\theta$ & $\begin{array}{l}m \\
\ddot{m} \\
\ddot{e}\end{array}$ & $\infty$ & 함 & $\bar{\sigma}$ & 每 & @̊ & $m$ & \\
\hline & 吾 & $\begin{array}{l}\stackrel{\complement}{\varpi} \\
\stackrel{\Xi}{\Sigma}\end{array}$ & $\underset{\infty}{+}$ & $\begin{array}{c}\widetilde{\Xi} \\
\stackrel{\infty}{\infty}\end{array}$ & $\stackrel{?}{\rightleftharpoons}$ & 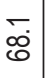 & $\overline{\dot{m}}$ & $\widehat{\tilde{N}}$ & $\begin{array}{c}\text { No } \\
\stackrel{b}{q}\end{array}$ & $\bar{g}$ & 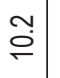 & ֶֻ & & $\overline{\overline{\tilde{\sigma}}}$ & $\begin{array}{c}\stackrel{c}{\bar{\varpi}} \\
\stackrel{\mathbb{d}}{\Sigma}\end{array}$ & $\stackrel{\circ}{\infty}$ & 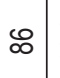 & \begin{tabular}{|l|}
$\infty$ \\
$\stackrel{0}{\circ}$ \\
$\stackrel{0}{\mid}$
\end{tabular} & $\begin{array}{l}\infty \\
\stackrel{\mathbb{T}}{\sim}\end{array}$ & $\underset{\check{\varpi}}{\approx}$ & ন & $\hat{g}$ & مْ & 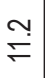 & 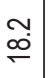 & \\
\hline & & ta & $\mathscr{c}$ & $\stackrel{\mathscr{E}}{E}$ & 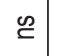 & $\stackrel{\mathscr{D}}{\boldsymbol{c}}$ & $\mathscr{c}$ & 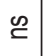 & $\stackrel{0}{\simeq}$ & $\mathscr{\simeq}$ & $\begin{array}{l}\text { O্ } \\
\dot{O} \\
0\end{array}$ & $\stackrel{\infty}{c}$ & & \pm & & $\mathscr{c}$ & $\stackrel{\mathscr{c}}{\simeq}$ & $\mathscr{c}$ & $\begin{array}{l}\infty \\
0 \\
0 \\
0 \\
0\end{array} \mid$ & $\stackrel{0}{\simeq}$ & $\mathscr{c}$ & $\stackrel{2}{\simeq}$ & $\stackrel{\infty}{2}$ & 2) & $\sum$ & \\
\hline$\frac{\mathscr{E}}{\sigma}$ & $\underset{T}{E}$ & क) & $\hat{\sim}$ & $\ddot{m}$ & ஸొ & $\stackrel{\infty}{\sim}$ & $\widehat{\sim}$ & $\mathscr{8}$ & $\begin{array}{l}\sigma_{0} \\
\infty\end{array}$ & $\stackrel{\nabla}{\dot{m}}$ & นี & $\begin{array}{l}\infty \\
\infty \\
\infty\end{array}$ & $\frac{\mathscr{d}}{\sigma}$ & $\underset{\text { II }}{\mathbb{f}}$ & क & $\stackrel{\infty}{\sim}$ & $\begin{array}{l}\text { g. } \\
\dot{j}\end{array}$ & ळ. & $\begin{array}{l}0 \\
\stackrel{\sim}{\sim}\end{array}$ & $\stackrel{?}{\sim}$ & ঃ & $\underset{\infty}{\sim}$ & ठ্ं. & $\stackrel{m}{6}$ & $\stackrel{\infty}{\stackrel{\circ}{\odot}}$ & \\
\hline एँ & $\begin{array}{l}\text { 莕 } \\
\Lambda 1\end{array}$ & 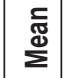 & $\approx$ & 守 & $\stackrel{\stackrel{\sim}{\rightleftharpoons}}{\rightleftharpoons}$ & $\widetilde{\widetilde{R}}$ & $\mid$ & $\bar{\sim}$ & $\begin{array}{l}\stackrel{0}{0} \\
\stackrel{y}{y}\end{array}$ & $\begin{array}{l}\circ \\
\stackrel{0}{q}\end{array}$ & 总 & 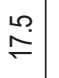 & 这 & $\begin{array}{l}\text { L } \\
\text { 人ิ }\end{array}$ & $\begin{array}{l}\stackrel{c}{\mathbb{E}} \\
\stackrel{\mathbb{d}}{\Sigma}\end{array}$ & $\stackrel{\wedge}{\infty}$ & \begin{tabular}{l|l} 
\\
$\stackrel{1}{\infty}$ \\
$\infty$ \\
$\infty$
\end{tabular} & هُ & 尊 & $\underset{\check{ల}}{\check{~}}$ & $\stackrel{\circ}{\sim}$ & $\begin{array}{l}\infty \\
\dot{q} \\
\dot{q}\end{array}$ & 宲 & $\stackrel{\text { مُ }}{\stackrel{\rho}{F}}$ & $\stackrel{2}{\square}$ & \\
\hline & $\begin{array}{l}\widetilde{6} \\
\stackrel{1}{1} \\
= \\
\end{array}$ & கి & $\stackrel{m}{\oplus}$ & $\widehat{\mathbb{J}^{*}}$ & $\stackrel{\mid}{\sim}$ & ז্ল & $\bar{\sigma}$ & ஜ & ఠ) & 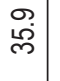 & $\stackrel{\text { مִ }}{\sim}$ & $\stackrel{m}{\sim}$ & & 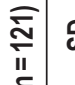 & क & $\underset{\sim}{\sim}$ & $\begin{array}{l}\text { L } \\
\text { | }\end{array}$ & $\stackrel{\sim}{\mathcal{F}}$ & 芯 & ஓे & 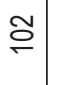 & 은 & 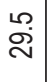 & مُ & $\bar{\sigma}$ & \\
\hline & 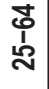 & $\mid \begin{array}{l}\text { व } \\
\stackrel{\mathbb{d}}{\Sigma}\end{array}$ & $\begin{array}{l}0 \\
\infty \\
\infty\end{array}$ & 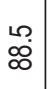 & 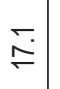 & : & 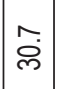 & ্ָ & 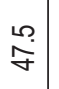 & 㤐 & ळ్ & 官 & & $\begin{array}{l}\text { S } \\
\vdots \\
\text { 心 }\end{array}$ & $\begin{array}{l}\underset{\mathbb{\varpi}}{\mathbb{d}} \\
\sum\end{array}$ & $\stackrel{m}{\infty}$ & $\begin{array}{l}\stackrel{\infty}{\infty} \\
\infty\end{array}$ & 앙 & $\begin{array}{l}\text {. } \\
\stackrel{2}{\circ}\end{array}$ & 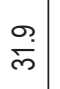 & $\widetilde{\widetilde{N}}$ & 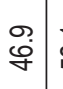 & స్తి & $\stackrel{\sim}{F}$ & $\stackrel{\infty}{\stackrel{1}{=}}$ & \\
\hline & 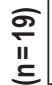 & 이 & $\widetilde{\infty}$ & 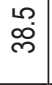 & $\stackrel{\sim}{\leftarrow}$ & 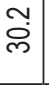 & $\begin{array}{l}0 \\
0 \\
0\end{array}$ & $\mathscr{8}$ & o & 守 & $\stackrel{\circ}{\sim}$ & $\bar{\sigma}$ & & 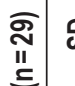 & क & $\check{F}$ & $\begin{array}{l}\mathscr{g} \\
\dot{\gamma}\end{array}$ & $\stackrel{\infty}{\infty}$ & $\hat{\tilde{g}}$ & مi & $\grave{I}$ & : & $\begin{array}{l}\infty \\
\dot{f}\end{array}$ & 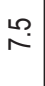 & $\underset{\infty}{\infty}$ & \\
\hline & $\begin{array}{c}\tilde{N} \\
\stackrel{\tilde{\omega}}{\sigma}\end{array}$ & 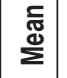 & $\stackrel{m}{\sim}$ & $\begin{array}{l}\stackrel{0}{0} \\
\stackrel{8}{2}\end{array}$ & $\stackrel{\oplus}{\stackrel{0}{\digamma}}$ & 莒 & $\mid \begin{array}{l}\infty \\
\stackrel{\sim}{\sim}\end{array}$ & $\stackrel{\circ}{\sim}$ & is & ठ̊. & $\begin{array}{l}\stackrel{R}{\hat{p}} \\
\stackrel{p}{p}\end{array}$ & $\stackrel{f}{\forall}$ & & $\begin{array}{l}\tilde{T} \\
\stackrel{0}{\sigma}\end{array}$ & $\begin{array}{l}\stackrel{c}{\mathbb{\varpi}} \\
\stackrel{\mathbb{d}}{\Sigma}\end{array}$ & 으 & $\begin{array}{l}\vec{\sigma} \\
\stackrel{\sigma}{*}\end{array}$ & $\begin{array}{l}\stackrel{\circ}{\circ} \\
\stackrel{0}{ }\end{array}$ & హี & $\stackrel{\mathscr{ల}}{\text { లె }}$ & $\underset{\sim}{\mathbb{N}}$ & 字 & ֶָ) & $\stackrel{\circ}{\circ}$ & $\begin{array}{l}\stackrel{\infty}{\infty} \\
\stackrel{\infty}{\circ}\end{array}$ & \\
\hline & 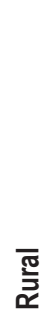 & & 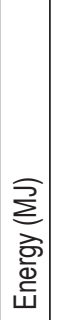 & 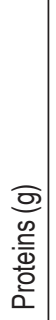 & 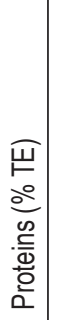 & 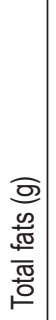 & 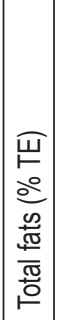 & 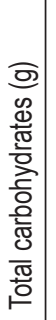 & 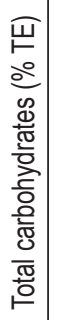 & 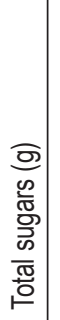 & 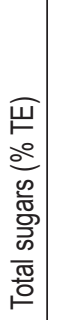 & 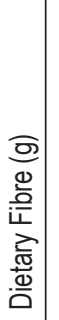 & & $\begin{array}{l}\text { 蛋 } \\
\text { ㄴ. }\end{array}$ & & 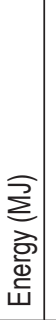 & 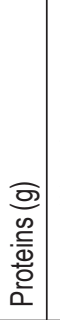 & 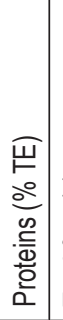 & 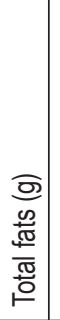 & 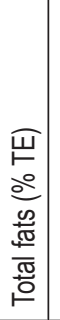 & 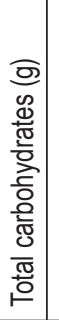 & 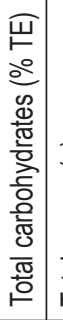 & 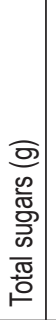 & 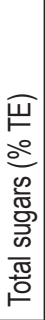 & 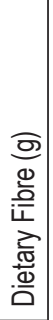 & 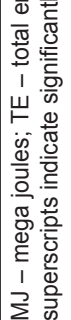 \\
\hline
\end{tabular}




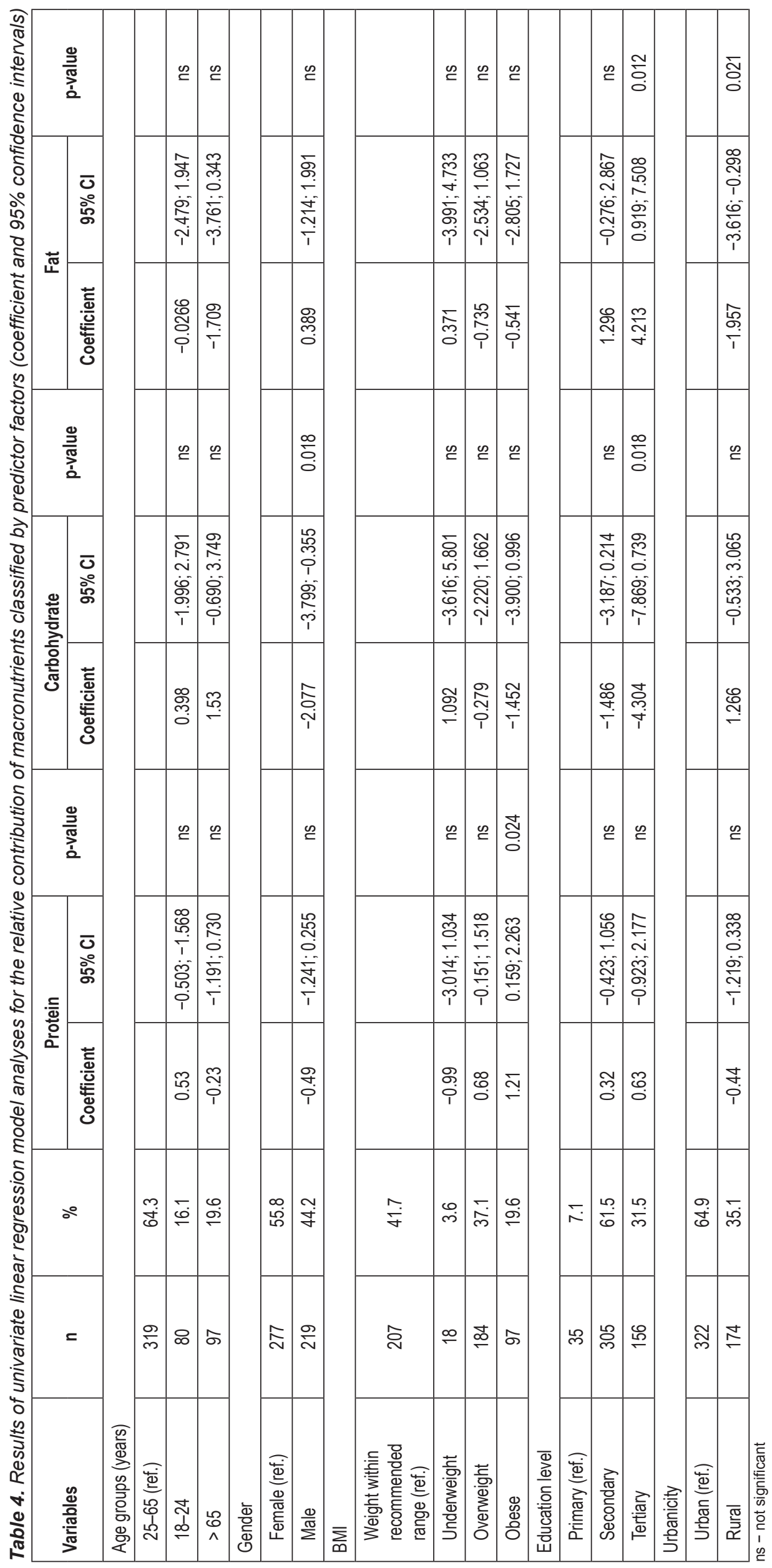


participants according to gender, we found, as expected, that males have significantly higher intake of energy, proteins, fats, carbohydrates and fibre than females. Level of significance was $<0.001$ for these variables. Females had significantly higher total sugar intake than males $(\mathrm{p}=0.042)$. Results were the same among urban participants but higher sugar intake among females was noticed for relative sugar intake $(\mathrm{p}<0.001)$. Differences in relative macronutrients intakes across age, gender, BMI groups, education levels, and type of settlement were assessed using univariate linear regression models (Table 4). There were no statistically significant differences in relative proteins intake between age, gender, education and type of settlement. Obese participants had significantly higher relative proteins intake comparing to participants with weight within recommended range $(p=0.024, \beta=1.21$, 95\% CI: $0.2-2.3)$. Unlike proteins, relative carbohydrates intake was significantly lower among males $(p=0.018, \beta=-2.077,95 \%$ CI: -3.8-3.4) as well as among participants with tertiary education comparing with those with primary one $(p=0.018, \beta=-4.304$, 95\% CI: $-7.9-0.7)$. For the relative fats intake, only education and type of settlement showed an impact. Participants with tertiary education had $4.2 \%$ higher relative fats intake comparing to those with primary education $(\mathrm{p}=0.012, \beta=4.213,95 \% \mathrm{CI}$ :
0.919-7.508), while participants from rural areas had significantly lower relative fats intake comparing to those living in urban settlements ( $p=0.021, \beta=-1.957,95 \%$ CI: $-3.616,-0.298)$. Inadequacy of macronutrients intake of the total sample according to each socio-demographic factor separately, as well as differences within factors, are shown in Figure 1. As shown there, the highest adherence to the national dietary guidelines was associated with consumption of proteins, with no significant differences in any of the examined socio-demographic factors. Still, low portions of primary educated (5.7\%) and underweight (5.6\%) subjects did not meet the national dietary recommendations. Carbohydrates are mostly consumed either according to the guidelines or below it. Primary educated participants had highest adherence to recommendations but also above it, and more than half of the obese participants consumed carbohydrates below national guidelines. Significantly higher adherence was observed among females than males $(p=0.018)$ and among urban comparing to rural participants $(\mathrm{p}=0.05)$. There was significantly higher adherence $(p=0.01)$ in carbohydrates intake of rural female participants $(62.4 \%)$ comparing to rural males $(39.5 \%)$. Adherence for fats was examined both for recommended intake according to the national guidelines and to WHO/FAO ones. Comparisons

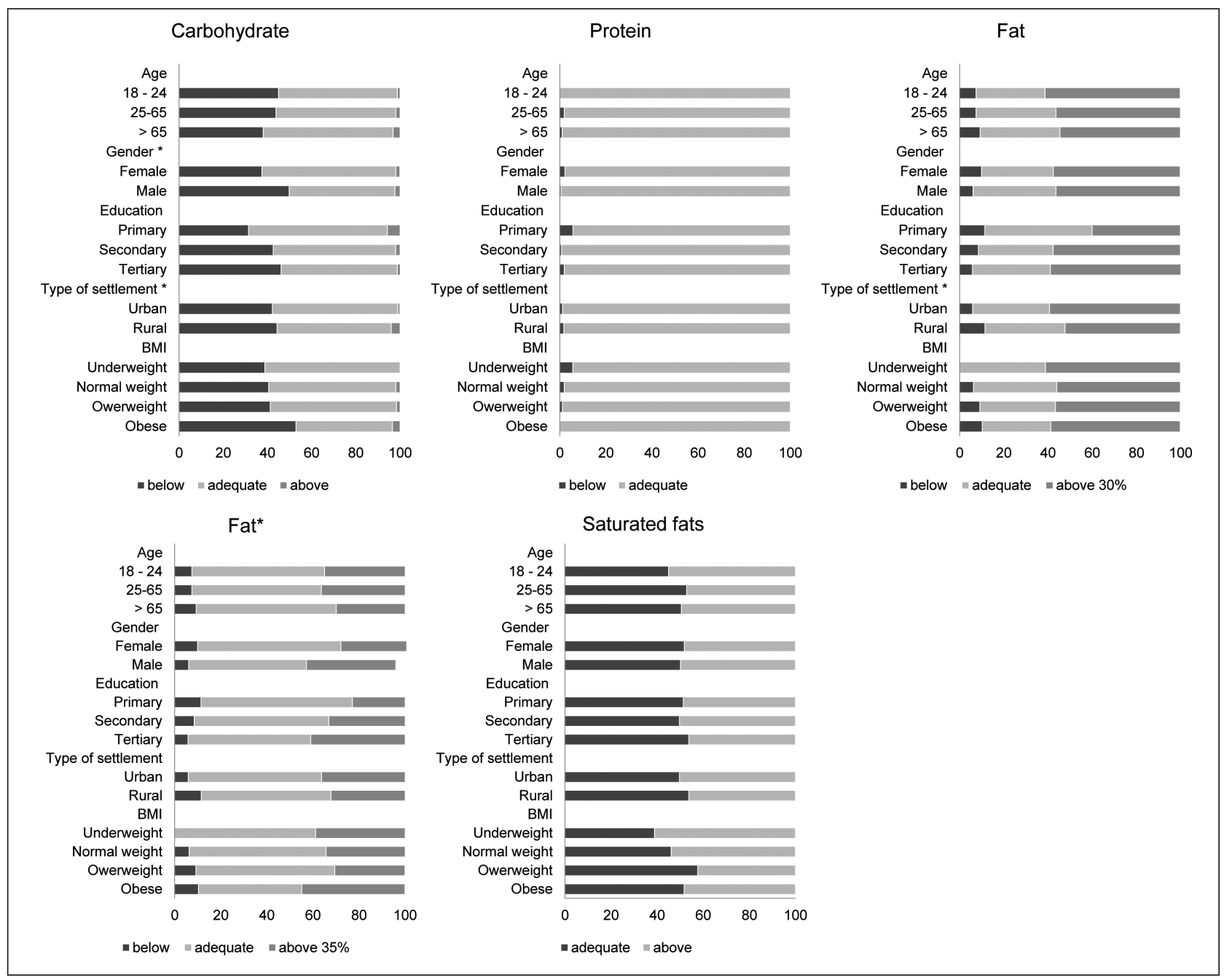

Fig. 1. Percentage of Macedonian adults with intake levels met, below and above the National Dietary Reference Intake (DRI) for macronutrients.

The $\mathrm{X}^{2}$ test was used to examine association between the three levels of meeting DRI and socio-demographic factors; a analysed according to the upper cut of value of $35 \%$ TE; ${ }^{*} p<0.05$ 


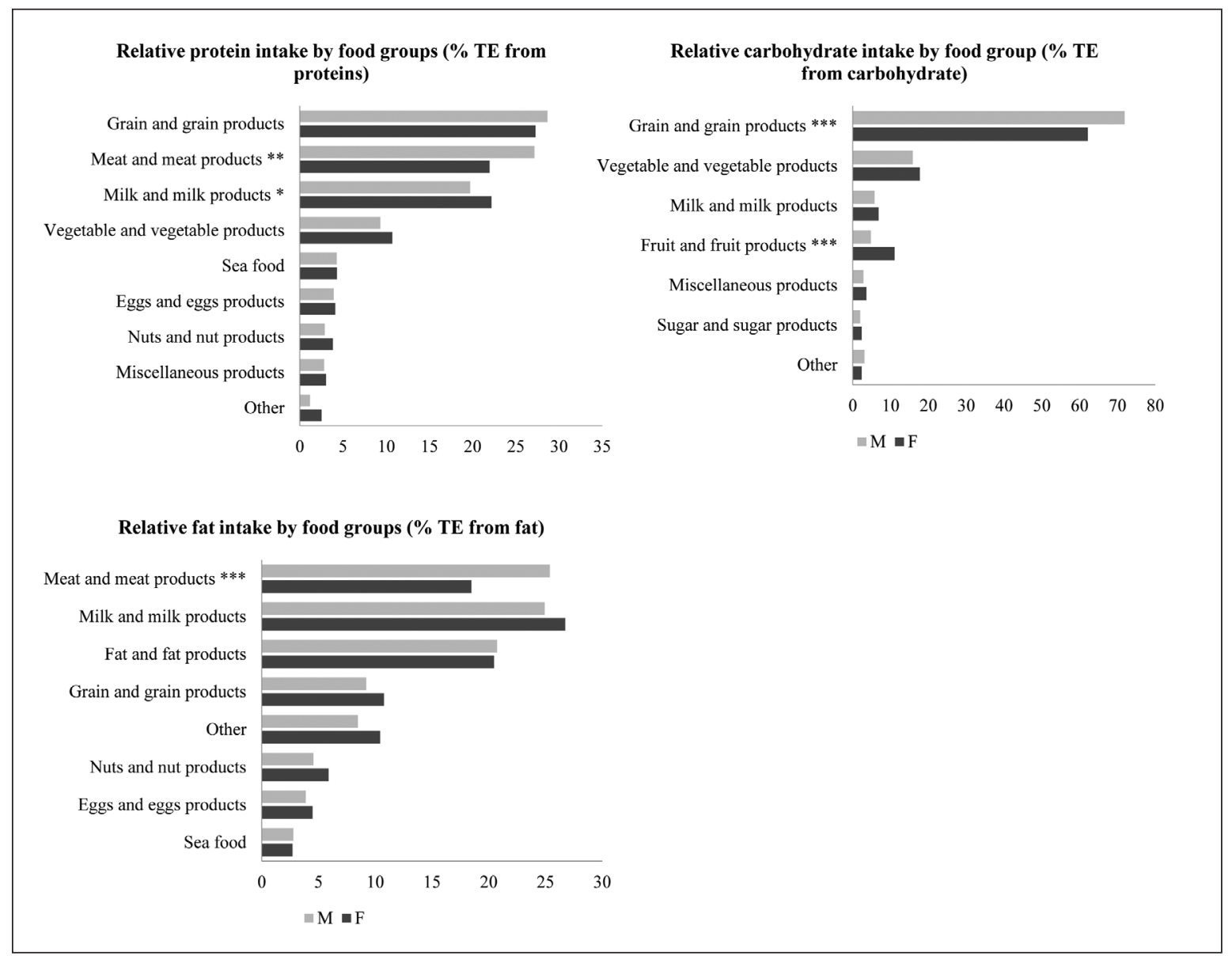

Fig. 2. Contributions of food groups to mean daily macronutrients intake of Macedonian adults according to gender. The $\mathrm{X}^{2}$ test was used to examine association between the three levels of meeting DRI and socio-demographic factors; ${ }^{* * *} \mathrm{p}<0.001 ;{ }^{* *} \mathrm{p}<0.01 ;{ }^{*} \mathrm{p}<0.05$

between and within the socio-demographic factors were similar, when different intake guidelines for fats were compared. However, there was significant difference between participants living in urban and rural settlement when adherence to fats consumption according to WHO/FAO guidelines was examined $(\mathrm{p}=0.05)$. The adherence to $\mathrm{WHO} / \mathrm{FAO}$ guidelines of the urban males over 65 was significantly higher comparing to other two age groups of urban males $(\mathrm{p}=0.05)$. We have also examined the consumption of saturated and other fatty acids and its adherence towards national dietary guidelines (Figure 1). As presented there, no significant difference existed in adherence to the recommended intake within any socio-demographic characteristic of the participants, with overweight subjects having the highest adherence to the recommended intake of saturated fats. When more thoroughly examined, the adherence for the saturated fatty acids among females aged 25-64 living in urban settlements was significantly higher compared to other two age groups of urban females $(p=0.004$, data not shown). The lowest level of adherence to the national dietary guidelines ( $>25 \mathrm{~g}$ for females, $>38 \mathrm{~g}$ for males) was seen in fibre. The lowest adherence was observed among urban males $(10.1 \%)$, which was significantly lower $(p=0.004)$ compared to urban females (21.7\%). Among age groups of urban participants, there was significantly higher adherence, within gender, in females aged $18-24(p=0.05)$ and males over 65 years of age $(p=0.03)$. Contributions of food groups to mean daily macronutrients intake of Macedonian adults, according to gender, is presented in Figure
2. Grain and grain products are primary source of carbohydrates in the diet of Macedonian adults. Statistically significant difference between genders was observed in grain's contribution in relative carbohydrates intake $(p<0.001)$. Female's consumption of fruits and fruit products contributed significantly more to the relative carbohydrates intake comparing to male's one $(p<0.001)$. Grains and meat were almost equally contributing as primary source of proteins in the diet. Meat and meat products were primary source of fats. Both for proteins and fats, there were statistically significant differences in relative meat intake in males and in females, as males consume more of these foods ( $p=0.003$ for proteins, $\mathrm{p}<0.001$ for fats).

\section{DISCUSSION}

Energy, macronutrients and fibre intake presented in this paper was observed in relation to age, gender and urbanization type of the participants. Males, both in urban and rural households, as confirmed in other research, had significantly higher intake of energy, proteins, fats, carbohydrates, and fibre compared to women (11). As we examined the effect of the age factor within the gender and type of urbanization, we saw the largest impact of age factor on urban males, since significant differences in total energy, proteins, fats and carbohydrates intake were observed among all age groups. Linear regression model implemented to 
assess the relative intake of macronutrients showed that there were no statistically significant differences in relative proteins intake between age, gender, education, and type of settlement. As expected, relative proteins consumption of men and obese participants was significantly higher than that of the participants with BMI within recommended range. Relative fats intake was higher among more educated participants and in those living in urban settlements. Intake of macronutrients in relation to education level and urbanicity has been explored in previous research and findings were different, with more recent ones showing lower fats intake among more educated consumers (12-14). We have assessed the adherence of macronutrients intake to dietary guidelines and the findings are similar with other research for all macronutrients $(15,16)$. Around half of the adults met the carbohydrates recommendations. This indicates that there is room for improvement, particularly in men and those living in rural settings, who should increase their carbohydrates intake. Fibre intake should be significantly increased, particularly in men below 65 years of age. This is important having in mind the evidence that intake of fibre is inversely associated with some types of cancer (17). Fats intake is frequently assessed in the dietary guidelines since it is related to the occurrence of cardiovascular diseases and other major NCDs. As expected, adherence to intake recommendations of fats was lowest compared to adherence to carbohydrates and proteins intakes, due to higher intake of fats. The nutritional transition that followed economic transition in MKD largely contributed to this phenomenon also described in research in other neighboring countries (18).

Premature mortality from major NCDs in MKD is higher compared to most countries in Europe (19). Previous research has proven that adherence to dietary guidelines may reduce the risk of premature and all-cause mortality (20-23). This makes strong case for public health nutrition professionals and decision makers to shape and implement policies that will promote adherence to dietary guidelines as important factor in improving health outcomes of the population. Data for contribution of food groups in daily macronutrients intake shows that grains, fruits and vegetables are primary source of carbohydrates in Macedonian population. It is interesting that gender difference is significant for grains and fruits, with women consuming more fruits and men grains. This is in accordance with previous research showing that women are the ones who take care for families to consume healthier foods (24). When it comes to meat and meat products, as less recommended foods and primary source of fats, males consume it significantly more than females. Results showed that meat and grain almost equally contribute to relative proteins intake of the adults in MKD. Even it is not common that meat and meat products alone are not primary source of proteins, we explain it with the high consumption of white bread in Macedonian population and the tradition to eat bread with every meal (25). This survey was also extremely important for obtaining data about anthropometric characteristics of the nutritional status since those data are not routinely collected for adults in the country. The rise of overweight and obesity are worldwide phenomenon and one of the most important public health issues of today (26). In MKD, overweight and obesity are more prevalent among males compared to females, as it is common in the countries of the nearest surrounding (27). The highest prevalence of overweight and obesity was observed among the elderly. This finding is in line with the similar ones in other research but we should be cautious with drawing conclusions since this does not necessary reflect the true age-adjusted difference (28). Obesity prevalence was the highest among primary educated participants and this finding confirms those in recent research concluding that there was larger increase in obesity prevalence in the low educational group, making education one of the main drivers of the increase of absolute inequalities in obesity in Europe over time (29-31). Newly adopted documents at European and global levels reconfirm the importance of nutrition and diet to prevent NCDs. MKD is aligning with those documents and actions but additional attention is needed in order to expect better health outcomes (32). There were, however, some limitations of the study. Those mostly apply to implementation of Computer Assisted Telephone Interview (CATI) for both 24-hour recalls. Although highly trained interviewers performed interviews by following specific protocol, this methodology did not allow using portion size measurement aids, like picture books, that may influence the accuracy of the reported quantities of foods consumed. However, these limitations do not affect the significance of the study results and the importance of data obtained towards NCDs prevention strategies.

\section{CONCLUSION}

This was the first population based and nationally representative study on food consumption among adult population in MKD. Its added value is the assessment of the prevalence of overweight and obesity among the adults in the country. The survey provides a detailed insight into the energy, macronutrients and fibre intake in relation to age, gender, education, and type of settlement of the household. The findings indicate that in MKD men are at greater risk of obesity related NCDs. Macronutrients intake of adults should be improved. There is higher intake of dietary fats and need for an increase of carbohydrates intake, with special focus on dietary fibre. Findings can be used in shaping, fine-tuning and implementing food and nutrition policies that will stimulate healthier diets as one of the major determinants in prevention of the diet related NCDs. It is expected that once established the food consumption surveys will run in regular time intervals in the future and for all population groups.

\section{Acknowledgements}

We gratefully thank all the participants who accepted to participate in the study for their cooperation, time and motivation. We particularly thank the WHO Country Office in MKD.

\section{Financial Support}

The work for this study was financially supported by the WHO Country Office in MKD with the Agreement for performance of work, WHO registration 2014/470701-0.

\section{Conflict of Interest}

None declared

\section{REFERENCES}

1. GBD 2015 Risk Factors Collaborators. Global, regional, and national comparative risk assessment of 79 behavioural, environmental and occupational, and metabolic risks or clusters of risks, 1990-2015: a systematic 
analysis for the Global Burden of Disease Study 2015. Lancet. 2016 Oct 8;388(10053):1659-724.

2. Mueller NT, Appel LJ. Attributing death to diet: precision counts. JAMA. 2017 Mar 7;317(9):908-9.

3. Institute for Health Metrics and Evaluation. Macedonia. What risk factors drive the most death and disability combined? [Internet]. Seattle: IHME [cited 2017 Aug 31]. Available from: http://www.healthdata.org/ macedonia.

4. Dietary guidelines for the population of the Republic of Macedonia [Internet]. Skopje: Institute of Public Health of the Republic of Macedonia; 2014 [cited 2019 May 26]. Available from: http://iph.mk/wp-content/ uploads/2014/11/nasoki ishrana-2014.pdf. (In Macedonian.)

5. Gurinović M. Nutrition epidemiology and public health nutrition. Reference Module in Food Science. 2016. doi: 10.1016/B978-0-08-1005965.03491-0.

6. Buzina R, Suboticanec K, Sarić M. Diet patterns and health problems: diet in southern Europe. Ann Nutr Metab. 1991;35 Suppl 1:32-40.

7. Republic of Macedonia, State Statistical Office. Census of population, households and dwellings in the Republic of Macedonia, 2002 - book XIII [Internet]. Skopje: State Statistical Office; 2005 [cited 2019 May 13]. Available from: http://www.stat.gov.mk/Publikacii/knigaXIII.pdf. (In Macedonian.)

8. Gurinović M, Milešević J, Novaković R, Kadvan A, Djekić-Ivanković M, Šatalić Z, et al. Improving nutrition surveillance and public health research in Central and Eastern Europe/Balkan Countries using the Balkan Food Platform and dietary tools. Food Chem. 2016 Feb 15;193:173-80.

9. Gurinović M, Milešević J, Kadvan A, Nikolić M, Zeković M, DjekićIvanković $\mathrm{M}$, et al. Development, features and application of DIET ASSESS \& PLAN (DAP) software in supporting public health nutrition research in Central Eastern European Countries (CEEC). Food Chem. 2018;238:186-94.

10. Diet, physical activity and cardiovascular disease prevention in Europe. Brussels: European Heart Network; 2011.

11. Heuer T, Krems C, Moon K, Brombach C, Hoffmann I. Food consumption of adults in Germany: results of the German National Nutrition Survey II based on diet history interviews. Br J Nutr. 2015;113(10):1603-14.

12. Petkeviciene J, Klumbiene J, Prättälä R, Paalanen L, Pudule I, Kasmel A. Educational variations in the consumption of foods containing fat in Finland and the Baltic countries. Public Health Nutr. 2007;10(5):518-23.

13. Spronk I, Kullen C, Burdon C, O'Connor H. Relationship between nutrition knowledge and dietary intake. Br J Nutr. 2014;111(10):1713-26.

14. Xu X, Byles JE, Shi Z, Hall JJ. Evaluation of older Chinese people's macronutrient intake status: results from the China Health and Nutrition Survey. Br J Nutr. 2015 Jan 14;113(1):159-71.

15. Harrington KE, McGowan MJ, Kiely M, Robson PJ, Livingstone MB, Morrissey PA, et al. Macronutrient intakes and food sources in Irish adults: findings of the North/South Ireland Food Consumption Survey. Public Health Nutr. 2001 Oct;4(5A):1051-60.

16. Ruiz E, Ávila JM, Valero T, del Pozo S, Rodriguez P, Aranceta-Bartrina $\mathrm{J}$, et al. Macronutrient distribution and dietary sources in the Spanish population: findings from the ANIBES study. Nutrients. 2016;8(3):177. doi:10.3390/nu8030177.

17. Bradbury KE, Appleby PN, Key TJ. Fruit, vegetable, and fiber intake in relation to cancer risk: findings from the European Prospective Investigation into Cancer and Nutrition (EPIC). Am J Clin Nutr. 2014 Jul;100 Suppl 1:394S-8S.
18. Ivanova L, Dimitrov P, Ovcharova D, Dellava J, Hoffman DJ. Economic transition and household food consumption: a study of Bulgaria from 1985 to 2002. Econ Hum Biol. 2006 Dec;4(3):383-97.

19. Global status report on noncommunicable diseases 2014. Geneva: WHO; 2014.

20. van Lee L, Geelen A, Kiefte-de Jong JC, Witteman JC, Hofman A, Vonk $\mathrm{N}$, et al. Adherence to the Dutch dietary guidelines is inversely associated with 20 -year mortality in a large prospective cohort study. Eur J Clin Nutr. 2016 Feb;70(2):262-8.

21. Struijk EA, May AM, Wezenbeek NLW, Fransen HP, Soedamah-Muth $\mathrm{SS}$, Geelen A, et al. Adherence to dietary guidelines and cardiovascular disease risk in the EPIC-NL cohort. Int J Cardiol. 2014;176(2):354-9.

22. Russell J, Flood V, Rochtchina E, Gopinath B, Allman-Farinelli M, Bauman A et al. Adherence to dietary guidelines and 15-year risk of all-cause mortality. Br J Nutr. 2013 Feb 14;109(3):547-55.

23. Germain L, Latarche C, Kesse-Guyot E, Galan P, Hercberg S, Briançon S. Does compliance with nutrition guidelines lead to healthy aging? A quality-of-life approach. J Acad Nutr Diet. 2013 Feb;113(2):228-40.

24. Spiroski I. Public health aspects of knowledge, attitudes and practices of the functional foods consumers in the Republic of Macedonia [Internet]. Skopje: Ss. Cyril and Methodius University in Skopje [cited 2019 May 13]. Available from: http://www.medf.ukim.edu.mk/upload/ dok/3095 142168635.pdf. (In Macedonian.)

25. Macedonia national nutrition survey 2011 among women of reproductive age and children 6-59 months of age [Internet]. Skopje: Institute of Public Health of the Republic of Macedonia; 2011 [cited 2019 May 13]. Available from: https://www.unicef.org/northmacedonia/media/2971/ file/MK 2011 NutritionSurvey ENG.pdf.

26. GBD 2015 Obesity Collaborators. Health effects of overweight and obesity in 195 countries over 25 years. N Engl J Med. 2017 Jul 6;377(1):13-27.

27. World Health Organization. Global Health Observatory (GHO) data. Overweight and obesity [Internet]. Geneva: WHO [cited 2017 Jan 1]. Available from: http://www.who.int/gho/ncd/risk_factors/overweight/en/.

28. Blundell JE, Baker JL, Boyland E, Blaak E, Charzewska J, De Henauw S, et al. Variations in the prevalence of obesity among European countries, and a consideration of possible causes. Obes Facts. 2017;10(1):25-37.

29. Loring B, Robertson A. Obesity and inequities: guidance for addressing inequities in overweight and obesity [Internet]. Copenhagen: WHO; 2014 [cited 2019 May 13]. Available from: http://ec.europa.eu/health/ social_determinants/docs/policybrief_obesity_en.pdf.

30. Gallus S, Odone A, Lugo A, Bosetti C, Colombo P, Zuccaro P, et al Overweight and obesity prevalence and determinants in Italy: an update to 2010. Eur J Nutr. 2013 Mar;52(2):677-85.

31. Hoffmann K, De Gelder R, Hu Y, Bopp M, Vitrai J, Lahelma E, et al. Trends in educational inequalities in obesity in 15 European countries between 1990 and 2010. Int J Behav Nutr Phys Act. 2017;14(1):63. doi: 10.1186/s12966-017-0517-8

32. Lai T, Stachenko S, Kostova NM, Ristovska G, Spiroski I. Better noncommunicable disease outcomes: challenges and opportunities for health systems The former Yugoslav Republic of Macedonia country assessment. Copenhagen: WHO; 2015.

Received March 20, 2018 Accepted in revised form September 12, 2019 